\title{
Imaging appearance of the involved mesenteric node in patient with systemic amyloidosis: A case report
}

Sunpob Cheewadhanaraks, M.D.(1)

Thitithep Suriyamonthon, M.D. ${ }^{(1)}$

Paramee Noisri, M.D. ${ }^{(1)}$

Pimporn Puttawibul, M.D. ${ }^{(1)}$

Tanawat Pattarapuntakul, M.D. ${ }^{(2)}$

Naruemon Wisedopas, M.D. ${ }^{(3)}$

Pakorn Arunsawat, M.D. ${ }^{(4)}$

Khanin Khanungwanitkul, M.D. ${ }^{(1)}$

From ${ }^{(1)}$ Department of Radiology, Faculty of Medicine, Prince of Songkla University,

Songkhla, Thailand.

${ }^{(2)}$ Department of Internal Medicine, Faculty of Medicine, Prince of Songkla University, Songkhla, Thailand.

(3) Department of Pathology, Faculty of Medicine, Chulalongkorn University, Bangkok, Thailand.

(4) Anatomical pathology unit, Hatyai Hospital, Songkhla, Thailand.

Address correspondence to K.K. (e-mail: khanin14@gmail.com)

Received 8 December 2020; revised 15 December 2020; accepted 16 December 2020

doi:10.46475/aseanjr.v21i3.98

\section{Abstract}

Amyloidosis is a rare disease characterised by abnormal amyloid protein deposition within the affected tissue. About $37 \%$ of the patients were presented with systemic amyloidosis, of which hilar, mediastinal, and para-aortic lymph nodes were involved. Deposition of amyloid protein in the mesenteric lymph node is rarely documented, but when reported, it is seen in isolated or secondary amyloidosis. Despite an indistinguishable imaging appearance of the amyloiddeposit mesenteric node from malignancy, infection, and an inflammation process, the radiologists should be aware of variable imaging findings to be suspicious of amyloidosis. We reported a rare case of systemic amyloidosis with mesenteric node involvement, manifested as node enlargement. 
Keywords: Amyloidosis, Mesenteric, Lymph node, Imaging findings, Clinical presentation.

\section{Introduction}

Amyloidosis is a rare group of disease defined by extracellular deposition and accumulation of abnormal proteins called amyloid. The most common form of this rare disease is systemic amyloidosis which is about $80-90 \%$ of all patients. Systemic amyloidosis can be classified into two groups based on biochemical structure of amyloid fibrils: primary amyloidosis (AL-amyloidosis) and secondary amyloidosis (AA-amyloidosis). AL-amyloidosis has a propensity to be associated with plasma cell disorders, especially multiple myeloma and monoclonal gammopathy. On the other hand, AA-amyloidosis is generally a result of infection or an inflammatory process such as osteomyelitis, granulomatous infection, connective tissue diseases, and neoplasms[1,2].

An imaging appearance of systemic amyloidosis can be varied and involve many organ systems such as cardiopulmonary, gastrointestinal, genitourinary, and musculoskeletal systems[3]. Additionally, lymph node involvement is not uncommon; about $37 \%$ of the patients demonstrated hilar, mediastinal, and para-aortic adenopathy[4]. On the contrary, the involvement of the mesenteric lymph node by amyloidosis is rather rare and seldom documented[5-6].

In this article, we would like to share a case report of systemic amyloidosis with pathologically proven mesenteric node involvement, which was accidentally revealed when the patient was referred for chronic diarrhea evaluation. Additionally, we also review a few case reports which published quite similar imaging appearances of this rare manifestation. 


\section{Case summary}

A 33-year-old woman, with an underlying hemoglobin E-beta thalassemia trait, was presented to the hospital with paraumbilical pain and chronic watery diarrhea, a large number of feces, about 500-1,000 mL each time without blood or mucous. The frequency is $4-5$ times per day, for 3 months. Her vital signs were unremarkable. The physical examination showed pale conjunctiva, a distended abdomen with tympanic on percussion and a hyperactive bowel sound without tenderness, nor a palpable mass. The laboratory results showed mild anemia (Hb 9.3 g/dL, Hct 30.1\%), hypoalbuminemia /hypogloglobuminemia (Alb 1.69 $\mathrm{g} / \mathrm{dL}$, Globulin $2.1 \mathrm{~g} / \mathrm{dL}$ ), hypokalemia and hyponatremia. The stool examination and stool acid fast strain were unremarkable. The initial bedside abdominal ultrasonography revealed a $5.3-\mathrm{cm}$ intraabdominal mass below the umbilicus. Therefore, subsequent computed tomography (CT) of the whole abdomen was performed (Figure 1).

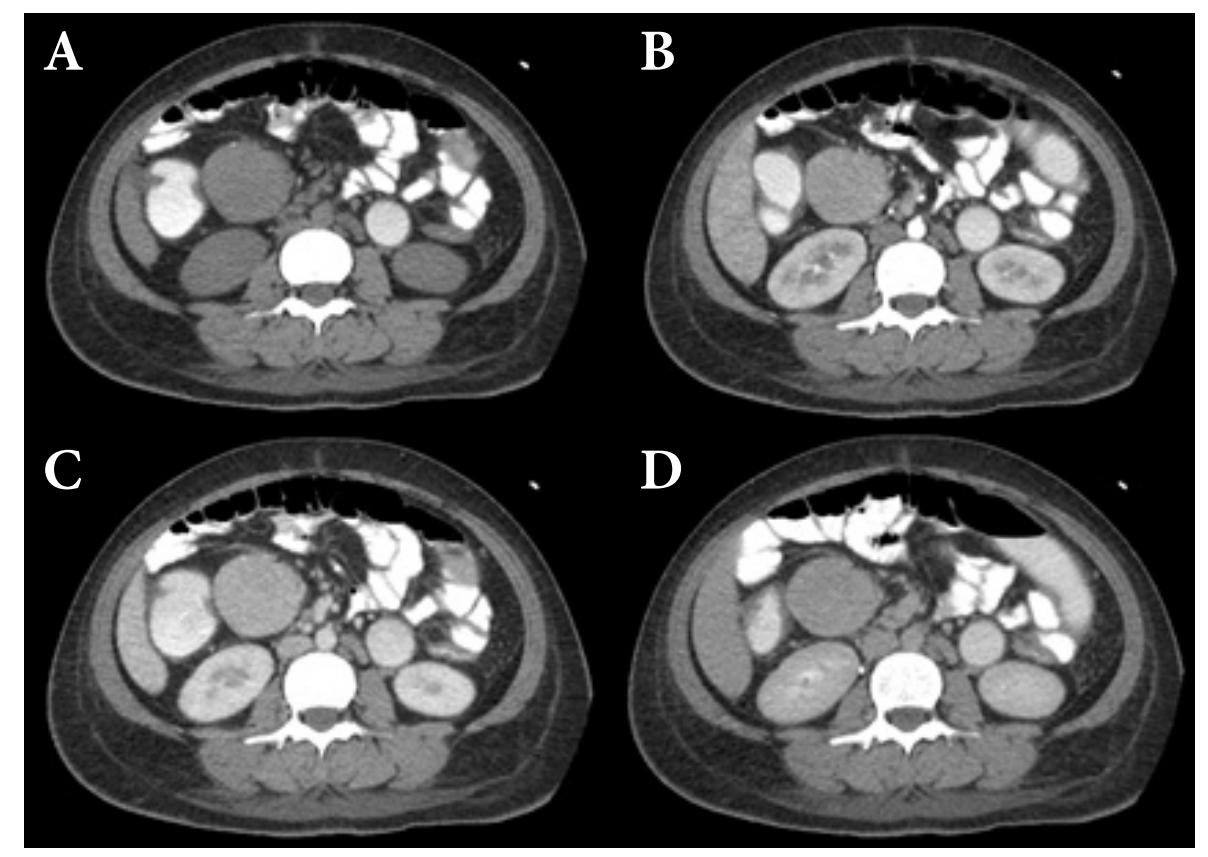

Figure 1. CT whole abdomen in plain $(A)$, arterial $(B)$, venous $(C)$ and delayed $(D)$ phases at 5 minutes shows a well-defined homogeneous mass with moderate enhancement at the right side of the mid abdomen. No intralesional calcification or necrosis is found. 


\section{Imaging, endoscopic, and pathologic findings}

She was admitted for supportive treatment and additional colonoscopy and esophagogastroduodenoscopy (EGD). The colonoscope was passed up to 10 $\mathrm{cm}$ from the ileocecal valve, which revealed ileitis and multiple small erosions. The EGD also showed diffuse erythematous mucosa without an ulcer along the stomach, duodenum, jejunum, and ileum. No obvious intraluminal mass was found along the gastrointestinal tract. She was treated as having colitis with intravenous antibiotics for 7 days. Then she was discharged after her clinical condition had been minimally improved.

However, after a few weeks, she was re-admitted due to acute paraumbilical pain with persistent chronic watery diarrhea and malnutrition. The physical examination still showed pale conjunctiva and mild tender at an umbilical area. Persistent hypoalbuminemia and diarrhea were suggestive of protein losing enteropathy. The technetium-99m human serum albumin abdominal scintigraphy was then performed (Figure 2). The result showed extravasation of the radiopharmaceuticals into the small bowel which confirmed protein losing enteropathy.

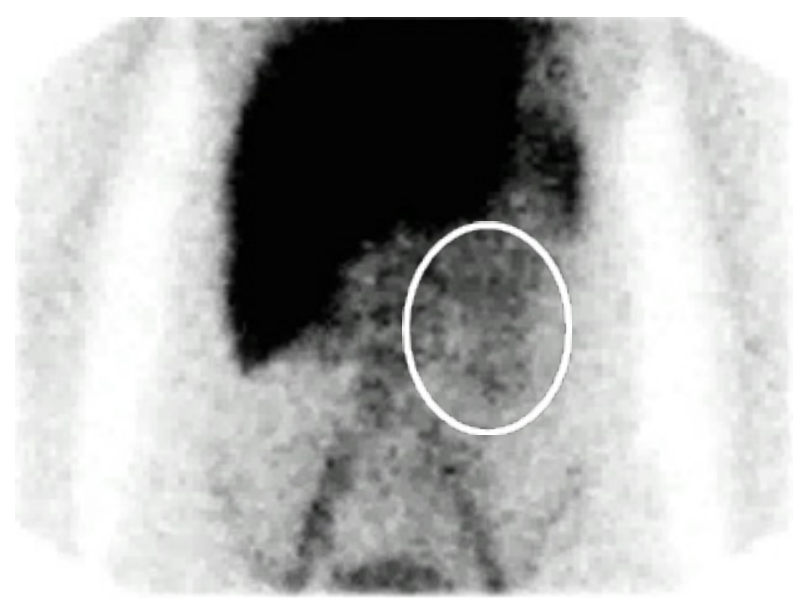

Figure 2. Abdominal scintigraphy was performed by dynamic study at 1 hour after injection of Technetium-99m labeled to human serum albumin ( ${ }^{99 m} \mathrm{Tc}-\mathrm{HSA}$ ). There is extravasation of radionuclides to the small bowel in the left upper quadrant with peristalsis in the dynamic study (white circle). Protein losing enteropathy at the small bowel area was suspected by this study. 
The follow-up CT of the whole abdomen showed insignificant change in size of the mass. Later on, the patient was scheduled for laparoscopic mesenteric mass resection. After the removal of the mass, the microscopic examination was performed (Figure 3). The result exhibited amyloid deposits in the mesenteric lymph node, which were confirmed by apple-green birefringence under polarised light on Congo-red stain. The patient was diagnosed to have amyloidosis.

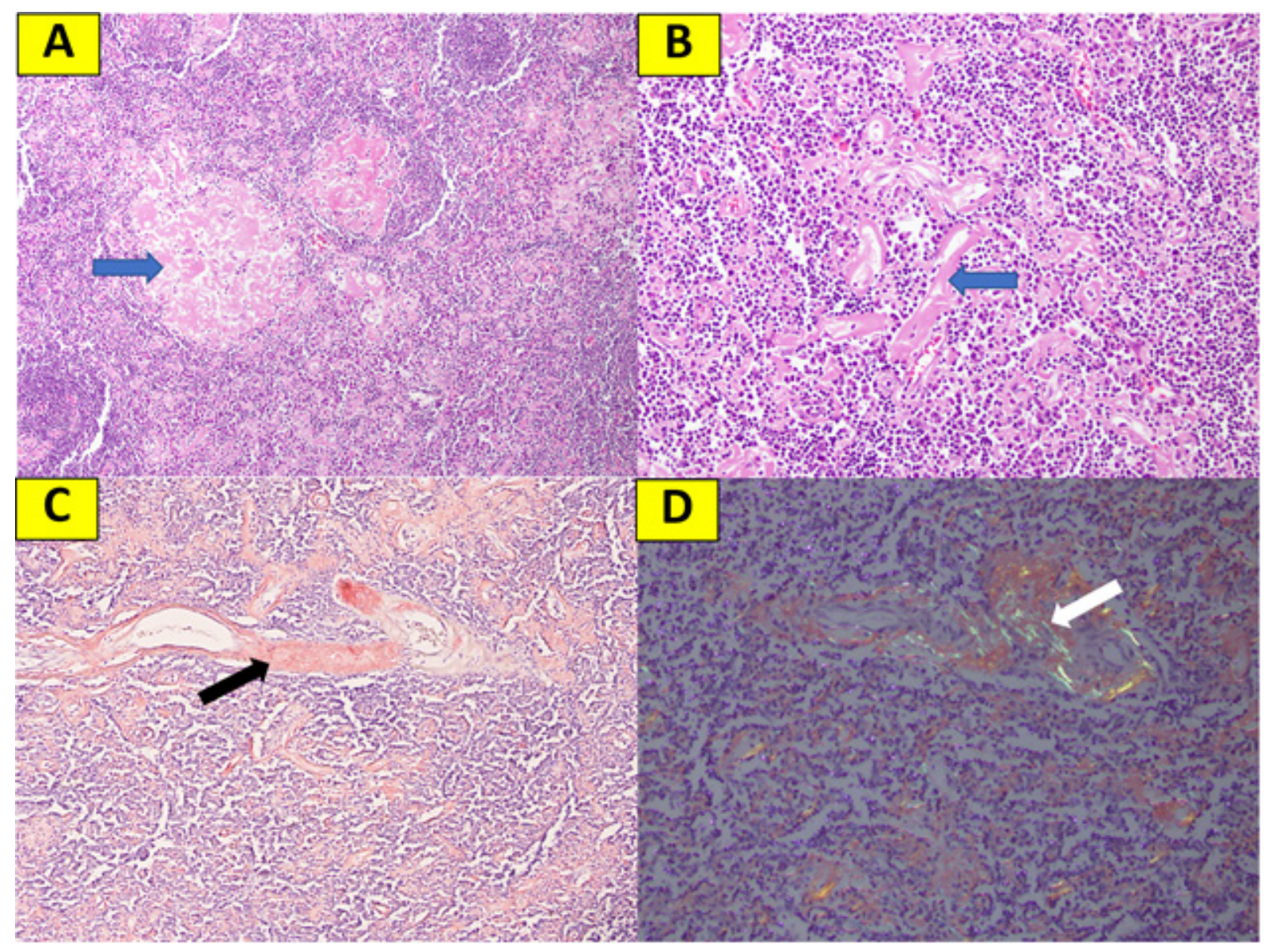

Figure 3. The microscopic examination of mesenteric mass resection demonstrated ( $A$ and B) abundant deposits of pink amorphous material (blue arrow) within the lymphoid tissue (Hematoxylin and Eosin); (C) amyloid deposits were confirmed by a positive Congo red stain, which revealed the characteristic salmon-pink color (black arrow) (Congo red); and (D) amyloid deposits exhibited characteristic apple-green birefringence under polarised light (white arrow) (Congo red under polarised light). 
Additional laboratory investigation including bone marrow biopsy, serum immunoelectrophoresis, and immunoglobulin free light chain were performed but all studies showed unremarkable results. However, the serum beta-2 microglobulin (B2M) was high. As a result, AA-amyloidosis is suggested as a final diagnosis rather than AL-amyloidosis.

After the mesenteric mass was removed by surgical excision, she was significantly improved in both watery diarrhea and malnutrition (anemia and edema). During the six-month follow-up after the surgery, she admittedly felt well and gained some weight. No additional treatment was given.

\section{Discussion}

Amyloidosis is a constellation of disease entities defined by extracellular deposition and accumulation of abnormal proteins called amyloid. The most common form is systemic amyloidosis, which could be either primary or secondary[1]. While hilar, mediastinal, and para-aortic nodes are commonly affected by this disease, the involvement of the mesenteric node is unarguably rare[4-6].

A few case reports, of which mesenteric nodes were involved, have claimed that amyloidosis-inflicted lymph nodes may contain intralesional calcification $[4,7,8]$. A recent case report by Bernabei et al.[8] revealed secondary amyloidosis associated with Castleman disease, which manifested as a large calcified mesenteric mass. Hiller at el. also claimed that internal punctate calcification can be found in $50 \%$ of amyloidosis-inflicted lymph nodes.

However, in our case with secondary amyloidosis, the involved mesenteric node was observed as a well-defined homogeneously enhanced mass without intralesional calcification. Bhavsar et al.[9] also published a case report of primary amyloidosis with multiple well-defined mesenteric masses showing homogeneously enhancement and absence of internal calcification, similar to our case. 
Therefore, we conclude that imaging features of lymph nodes are non-specific, whether they contain intralesional calcifications or not. If there is no intralesional calcification, the differential diagnosis includes hematologic malignancy, metastasis, infection, and an inflammatory process. Under different circumstances with intralesional calcification, the differential diagnosis includes ovarian cancer, granulomatous infection, primary carcinoid tumor, lymphoma after chemotherapy, and sclerosing mesenteritis[10].

Eventually, definite diagnosis by histology is required. The positive result is seen as an appearance of classic apple-green color birefringence under the polarised light, after staining the tissue with Congo red[11].

\section{Conclusion}

Mesenteric node involvement by systemic amyloidosis, manifested as an enlarged node, is rare but possible, as in our case and a few other case reports. Intralesional calcification could be a helpful feature for suggesting amyloidosis. When the intralesional calcification is absent, the radiologists should still be aware of variable imaging findings and possibility of amyloidosis. However, histological confirmation is unquestionably required for definite diagnosis. 


\section{References}

1. Ozcan HN, Haliloglu M, Sokmensuer C, Akata D, Ozmen M, Karcaaltincaba M. Imaging for abdominal involvement in amyloidosis. Diagn Interv Radiol 2017;23:282-5. doi: 10.5152/dir.2017.16484.

2. Scott PP, Scott WW Jr, Siegelman SS. Amyloidosis: an overview. Semin Roentgenol 1986;21:103-12. doi: 10.1016/0037-198x(86)90027-1.

3. Georgiades CS, Neyman EG, Barish MA, Fishman EK. Amyloidosis: review and CT manifestations. Radiographics 2004;24:405-16. doi: 10.1148/ rg. 242035114.

4. Vanhoenacker FM, Vanwambeke K, Jacomen G. Amyloidosis: an unusual cause of mesenteric, omental and lymph node calcifications. JBR-BTR 2014;97:283-6. doi: 10.5334/jbr-btr.1329.

5. Mohan V, Kemp JA, Lewine HE, Rabin M, Goldstein ML, Farraye FA. Diffuse mesenteric amyloidosis. Dig Dis Sci 1997;42:1079-82. doi: 10.1023/a:1018809724577.

6. Glynn TP Jr, Kreipke DL, Irons JM. Amyloidosis: diffuse involvement of the retroperitoneum. Radiology 1989;170(3 Pt1):726. doi: 10.1148/radiology. 170.3.2916026.

7. Bernabei L, Waxman A, Caponetti G, Fajgenbaum DC, Weiss BM. AA amyloidosis associated with Castleman disease. Medicine(Baltimore) 2020; 99: e18978. doi: 10.1097/MD.0000000000018978.

8. Hiller N, Fisher D, Shmesh O, Gottschalk-Sabag S, Dolberg M. Primary amyloidosis presenting as isolated mediastinal mass: diagnosis by fine needle biopsy. Thorax 1995;50:908-9. doi: 10.1136/thx.50.8.908. 
9. Bhavsar T, Vincent G, Durra H, Khurana JS, Huang Y. Primary amyloidosis involving mesenteric lymph nodes: diagnosis by fine-needle aspiration cytology. Acta Cytol 2011;55:296-301. doi: 10.1159/000324181.

10. Sheth S, Horton KM, Garland MR, Fishman EK. Mesenteric neoplasms: CT appearances of primary and secondary tumors and differential diagnosis. Radiographics 2003;23:457-73. doi: 10.1148/rg.232025081.

11. Howie AG, Brewer DB, Howell D, Jones AP. Physical basis of colors seen in Congo red-stained amyloid in polarised light. Lab Invest 2008;88:232-42. doi: 10.1038/labinvest.3700714. 